\title{
Differences in Emission Properties for Triboluminescent EuD4TEA Synthesized Using Europium Nitrate or Europium Acetate
}

\author{
William A. Hollerman ${ }^{1 *}$, Ross S. Fontenot ${ }^{2}$, and John Miller ${ }^{1}$ \\ ${ }^{1}$ University of Louisiana at Lafayette, Department of Physics, PO Box 44210, Lafayette, LA 70504, USA \\ 2 BAI, Inc., 5845 Richmond Hwy, Alexandria, VA 22303, USA \\ *e-mail: hollerman@louisiana.edu
}

\begin{abstract}
Europium tetrakis dibenzoylmethide triethylammonium $\left(\mathrm{EuD}_{4} \mathrm{TEA}\right)$ is one of the most intense triboluminescent (TL) materials currently known. It was recently reported that the triboluminescent yield was increased by $82 \%$ by changing the starting europium salt from europium chloride to europium nitrate. Unfortunately, europium nitrate has been classified as a hazardous material that cannot be shipped by air, which limits the availability of manufacturers and increases the cost. In an effort to further reduce the synthesis cost of $\mathrm{EuD}_{4} \mathrm{TEA}$, we explore the effects of europium acetate on the triboluminescent properties of EuD4TEA by comparing the triboluminescent yield. The photoluminescent spectra as well as crystal structure using an optical microscope will also be explored.
\end{abstract}

\section{Introduction}

Triboluminescence (TL) is the light emitted from a material when it undergoes fracture [1]. While this unique phenomenon has been known for over 400 years, no theory has yet to predict whether a material will emit TL. However, this has not stopped scientists from coming up with many unique applications. One such application is the use as the active element for impact and damage sensors [1-13]. For example, in 1999, Sage and Geddes used TL to patent a sensor capable of discerning the locations of an impact $[8,9,14]$. Their simple design involved coating a structure with a triboluminescent material or creating a composite triboluminescent object $[8,9,14]$. A sensor would then be embedded within the structure or mounted on its surface [14]. Impacts to the structure would produce light, which would be recorded and analyzed to determine the location [14]. In addition, Sage et al. proposed that several different triboluminescent materials could be used and arranged at various locations $[8,9,14]$. The advantage is that when an impact takes place, its location could be determined by the wavelength emitted [14]. For example, by placing two different triboluminescent materials at known distances from the detector, it is possible to determine the approximate location of the impact by measuring the emitted wavelength.

Unfortunately, the triboluminescent emission yield for most materials is small; therefore, the resulting light can be difficult to detect. Based on a recipe developed by Hurt et al. [15], Fontenot and Hollerman et al. have been synthesizing europium tetrakis dibenzoylmethide triethylammonium $\left(\mathrm{EuD}_{4} \mathrm{TEA}\right)$ for the last decade $[2,16]$. This material is bright enough to be seen in daylight and has $206 \%$ of the triboluminescent emission yield of $\mathrm{ZnS}$ :Mn when subjected to low energy impacts [2,17]. In fact, Fontenot et al. showed that adding dibutyl phosphate increases the triboluminescent emission yield by a factor of more than six compared to ZnS:Mn [18].

While emission from this material is very bright, it uses europium compounds as precursors, which are expensive in today's market. Just a few years ago, europium nitrate ( $99.999 \%$ pure) could be purchased for about \$3-5 per gram (not including shipping costs). Since the 1990s, a large fraction of rare earth elements (such as europium) have come from China. Since 2007, the Chinese government has restricted mining and production of rare earth elements to conserve resources and to give supply preference to their manufacturers. For this reason, the cost of compounds made with rare earth elements has greatly increased. Currently, the most economical europium nitrate $(99.9 \%$ pure) costs about $300 \%$ what it did a few years ago. Adding to this increase, europium nitrate is now considered to be a hazardous material (oxidizer) in the United States.

To reduce the cost of synthesizing EuD4TEA, we decided to use europium acetate instead of europium nitrate as the precursor material. Europium acetate was chosen because it can be dissolved in ethanol, it is the same europium ion state as common europium nitrate and is significantly less expensive. This paper will compare the TL properties of EuD4TEA made from both europium nitrate and acetate precursors. 


\section{Methods and Materials}

\section{Synthesis of EuD $D_{4} T E A$}

The synthesis of EuD4 4 TEA was based on procedures used in Reference [2]. The process began by pouring $25 \mathrm{~mL}$ of HPLC grade anhydrous denatured ethanol (Alfa Aesar, 22930) into an Erlenmeyer flask and placed onto a hotplate. Afterwards, $4 \mathrm{mmol}$ of 99.9\% (REO) europium (III) acetate hydrate (Alfa Aesar, 42878) was added to the hot solution. Then, $13 \mathrm{mmol}$ of $99 \%$ (GC) 1,3-diphenyl-1,3-propanedione (Chem-Impex, 00693) also known as dibenzoylmethane (DBM) was added. This addition caused the clear solution to turn dark yellow. Once the DBM was dissolved, the flask was removed from the hotplate and $14 \mathrm{mmol}$ of $\geq 99.5 \%$ triethylamine (TEA) (Sigma Aldrich, 471283) was added. The solution was then kept aside to cool at ambient temperature. Pictures of the just synthesized EuD 4 TEA solution made with europium acetate and europium nitrate are shown in Fig. 1(a) and 1(b) respectively. Note that both $\mathrm{EuD}_{4}$ TEA samples shown in Fig 1. were illuminated with ultraviolet (UV) light. The $\mathrm{EuD}_{4}$ TEA powder that formed were light yellow in color, clumpy, and hard as shown in Fig 2(a). The same procedure was then repeated using 99.9\% (REO) europium (III) nitrate hexahydrate (Alfa Aesar, 15290), which produced a light-yellow powder that was very sparkly as shown in Fig. 2(b).

(a)

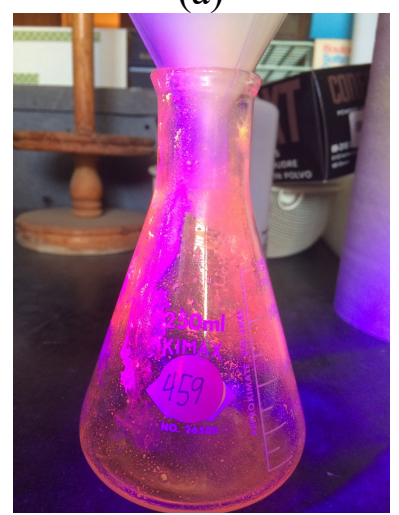

(b)

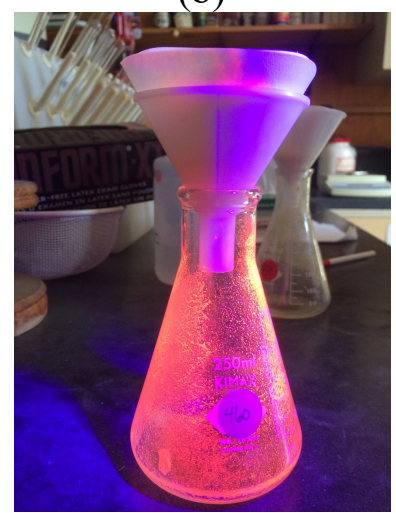

Fig. 1. Pictures of the just synthesized EuD 4 TEA solution made with (a) europium acetate and (b) europium nitrate. These samples were illuminated with UV light.

(a)

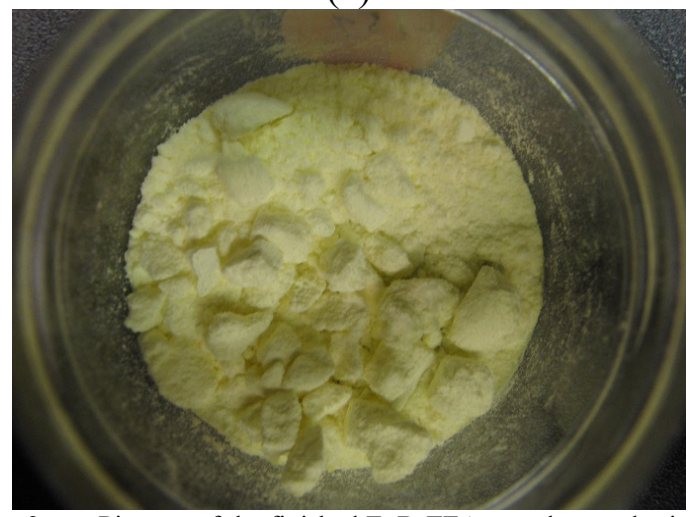

(b)

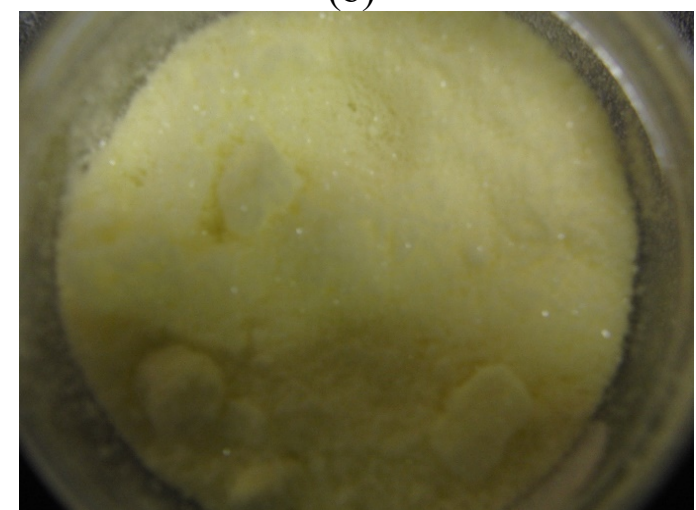

Fig. 2. Pictures of the finished $\mathrm{EuD}_{4} \mathrm{TEA}$ samples synthesized with (a) europium acetate and (b) europium nitrate. These pictures were taken under standard white room light.

\section{Triboluminescent Testing}

Once the crystals were completely dried, they were placed in a small clear round wide-mouth jar for storage. Using a custom built drop tower described in Reference [17], the crystalline products were tested for their triboluminescent properties. The measurement began by placing a $0.10 \mathrm{~g}$ of sample powder on the Plexiglass plate. The powder is arranged so that it is positioned around the center of the tube with a minimum height. A $130 \mathrm{~g}$ steel ball 
is positioned on a pull pin at a set distance of 42 inches $(1.1 \mathrm{~m})$ above the material. The pin is pulled and the ball falls and impacts the sample material producing TL. After each test, the drop tube is removed, the ball is cleaned, and the sample powder is redistributed near the center of the target area [17].

To determine the triboluminescent yield for a given sample, a United Detector photodiode is positioned under the Plexiglass plate $2.25 \mathrm{~cm}$ below the sample. A Melles Griot large dynamic range linear amplifier set to a gain of $20 \mu \mathrm{A}$ increases the signal amplitude. A Tektronix 2024B oscilloscope records the signal in single sequence mode with a $500 \mu \mathrm{s}$ measurement time. Once the signal is acquired, it is analyzed using custom LabVIEW program that integrates the area under the curve and calculates the decay time for the particular emission [17].

\section{Photoluminescent Testing}

Unlike fluorescence, phosphorescence utilizes both the singlet and triplet excited states [19]. The europium complex has an emission mechanism consisting of: the ligand absorbs energy, undergoes intersystem crossing into a triplet state, and then transfers its energy to the $\mathrm{Eu}^{3+}$ ion [19]. The phosphorescence of each material was excited using a 4 W $365 \mathrm{~nm}$ Raytech UV lamp (LS-7CB). It should be noted that each material was fresh, i.e., not tested for TL. The photoluminescence was recorded using an Ocean Optics USB 2000 spectrometer. The integration time was controlled using the SpectraSuite program and increased by the software until the photoluminescent emission spectrum reached its maximum that was just below the saturation limit of the spectrometer. Once this was determined, the dark background was subtracted and one hundred spectra were recorded and averaged.

\section{Light and Electron Microscopy}

To better understand the crystal structure, the samples were examined using a standard light microscope as well as a JOEL-6300FV field emission scanning electron microscope (SEM). The unused samples were first attached to metal stubs using carbon tape. Then they were gold coated so that they could be analyzed using the SEM. An Electron Microscopy Sciences 550x sputter coater was used to coat a $12 \mathrm{~nm}$ gold layer on each sample. Once the thin coats were dry, they were placed inside the SEM. The working distance, till, accelerating voltage, and emission current were then set to $22 \mathrm{~mm}, 300 \mathrm{~mm}, 15 \mathrm{kV}$, and $8 \mu \mathrm{A}$, respectively.

\section{Experimental Results}

Using the drop tower and LabVIEW VI tools described above, the effects of europium acetate on the triboluminescent properties of EuD 4 TEA is shown in Fig 3. Due to this being a relative measurement, all the data was normalized to the europium nitrate result. The error in normalized TL light yield was estimated to be $7 \%$ which is the error from synthesizing the material and the drop tower apparatus [20]. From these results, it is evident that europium acetate provides about half of the triboluminescent yield as the nitrate version. It should be noted that while the triboluminescent yields were different, the decay times are statistically identical. The measured triboluminescent decay time for the acetate based $\mathrm{EuD}_{4} \mathrm{TEA}$ was $522.7 \pm 10.1 \mu \mathrm{s}$, while the nitrate based $\mathrm{EuD}_{4} \mathrm{TEA}$ decay time was $524.3 \pm 17.7 \mu \mathrm{s}$.

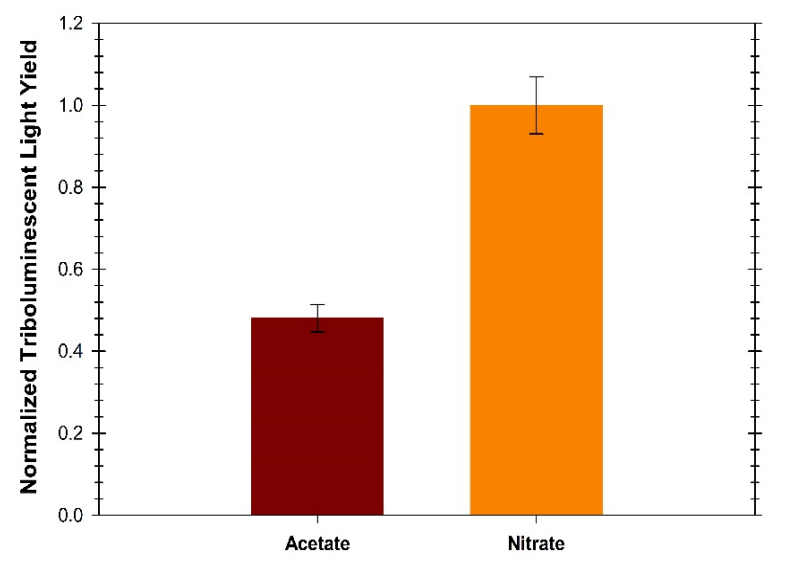

Fig. 3. Comparison of the TL produced by $\mathrm{EuD}_{4} \mathrm{TEA}$ synthesized with europium acetate and nitrate precursors. 
To better understand why the acetate provides a lower triboluminescent yield, we first used a standard optical microscope to investigate the crystals. As you can see in Fig. 4(a), the crystals formed by the acetate are cloudy. Meanwhile, the crystals produced by the nitrate are clear as shown in Fig. 4(c). This could be explained using the solubility. When acetate was used to create $\mathrm{EuD}_{4} \mathrm{TEA}$, the acetate had a very low solubility with ethyl alcohol (it was completely insoluble with acetone). This in turn caused a quick precipitation and rougher crystal. Conversely, the europium nitrate has a higher solubility with ethyl alcohol, which slows the precipitation and allows for a better crystal to form. This is verified by the SEM images shown in Figs. 5, 6, and 7. Fig. 5(a) and 6 show the acetate based EuD 4 TEA looks dirty, i.e., it is typically a rod shape with many smaller rods growing around it. Conversely, Fig. 5(b) and 7 show the nitrate based EuD 4 TEA tends to be more plate-like and cleaner in scope. More research is needed to fully understand these results.

(a)

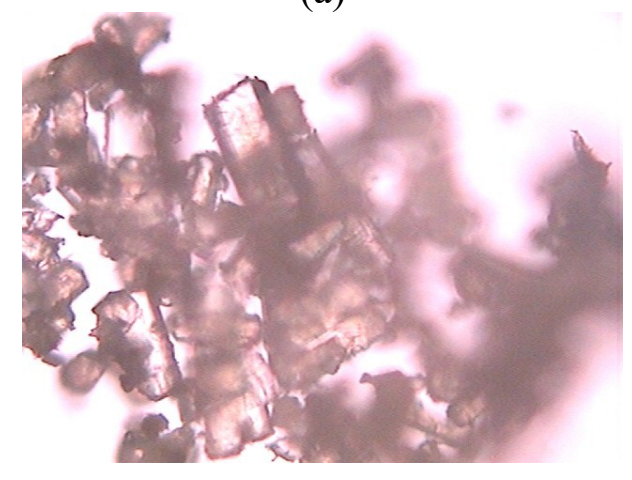

(c)

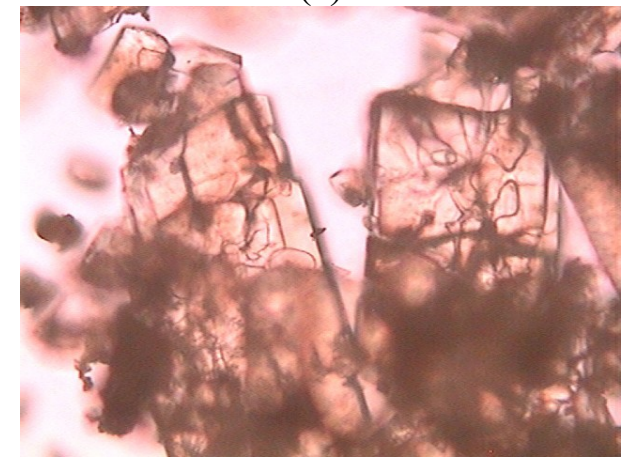

(b)

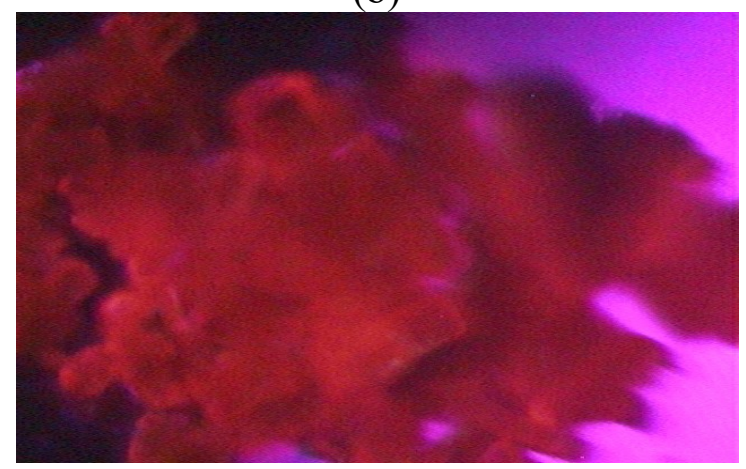

(d)

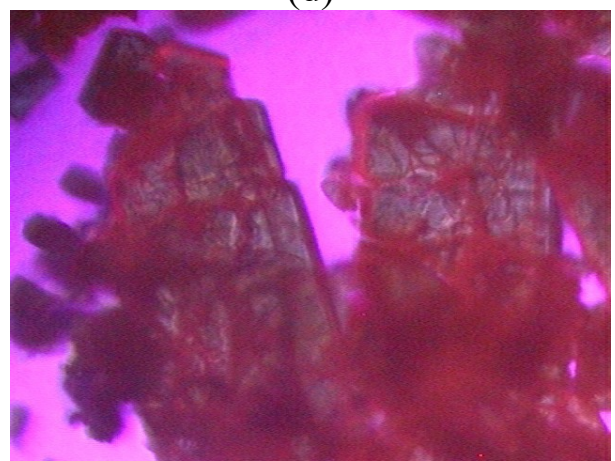

Fig. 4. Optical microscope pictures of EuD 4 TEA crystallites made with europium acetate and nitrate at a magnification of forty. EuD 4 TEA made with europium acetate is shown under (a) regular white light and (b) ultraviolet light. EuD 4 TEA made with europium nitrate is shown under (c) regular white light and (d) ultraviolet light. EuD4TEA

(a)

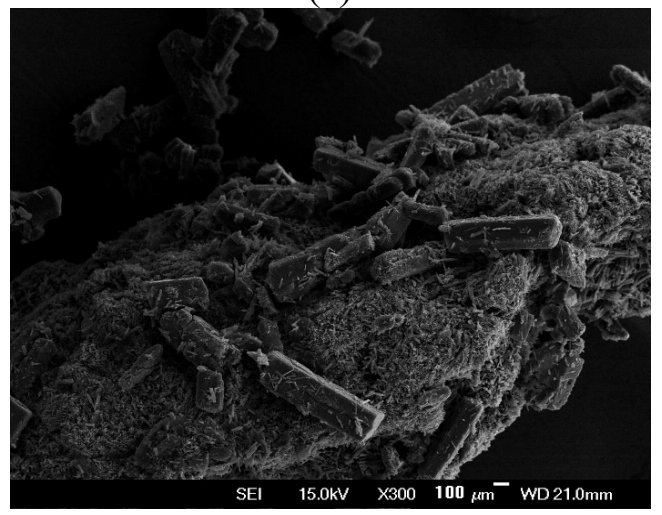

(b)

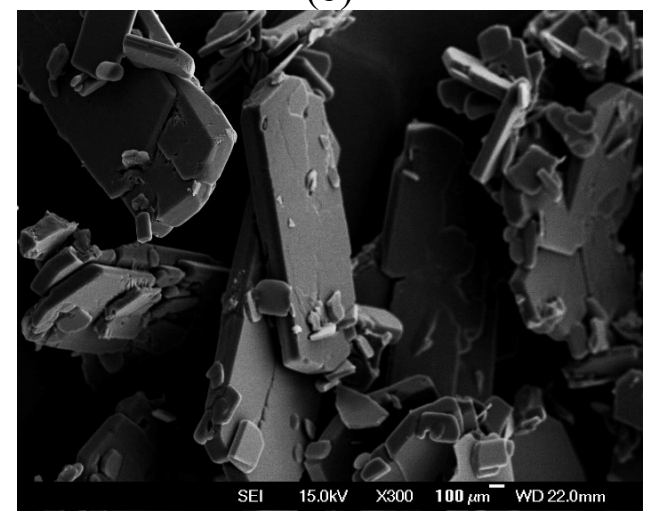

Fig. 5. Scanning electron microscope (SEM) images of EuD 4 TEA crystallites made with (a) europium acetate and (b) europium acetate precursors at a magnification of 300 . 


\section{$9,000 x$}

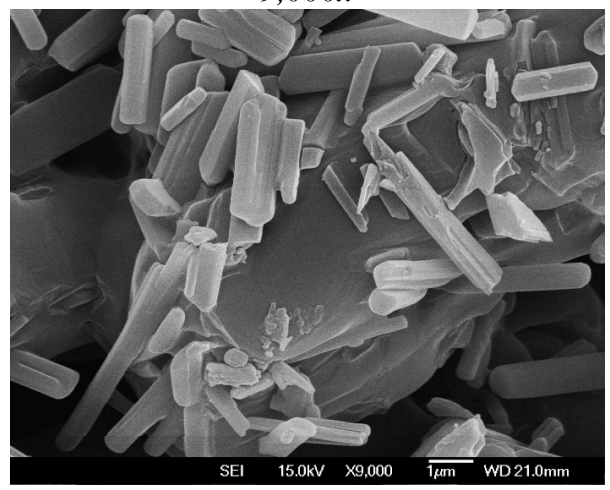

$20,000 x$

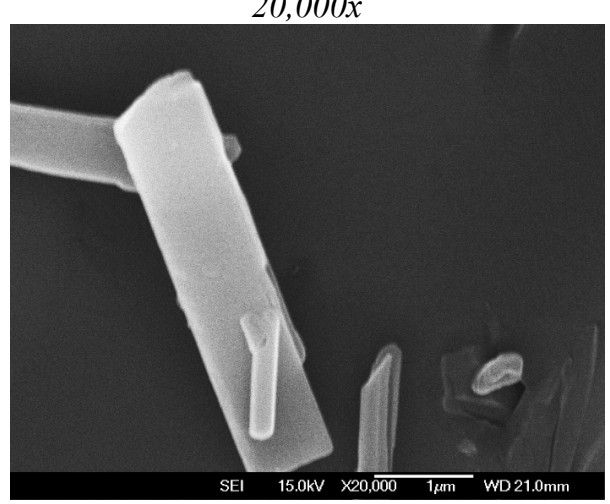

$3,000 x$

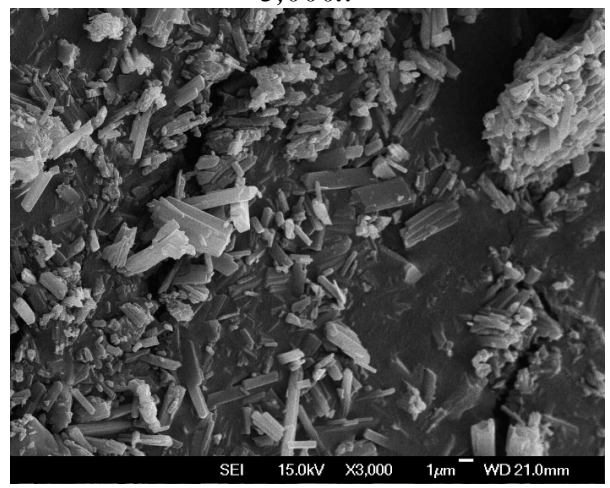

$300 x$

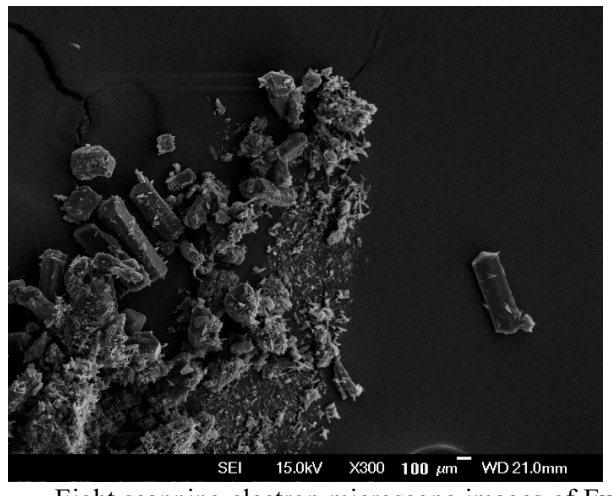

$20,000 x$

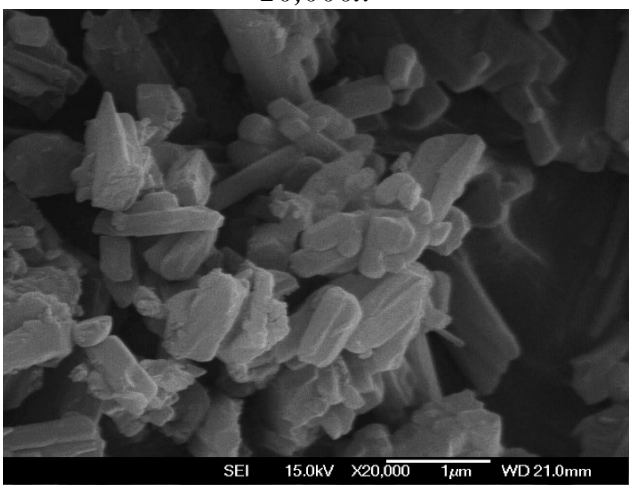

$1,600 x$

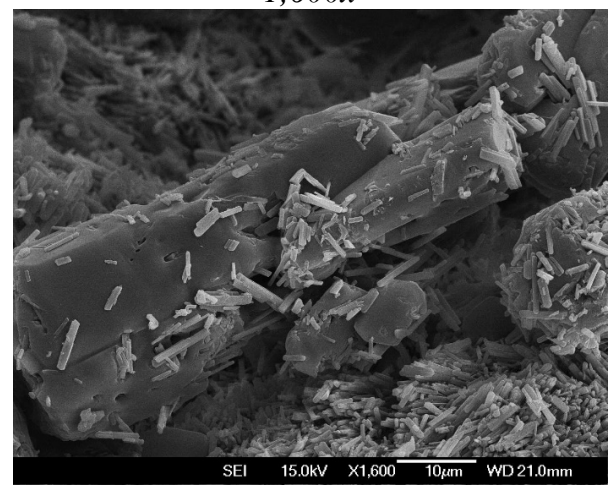

$1,000 x$

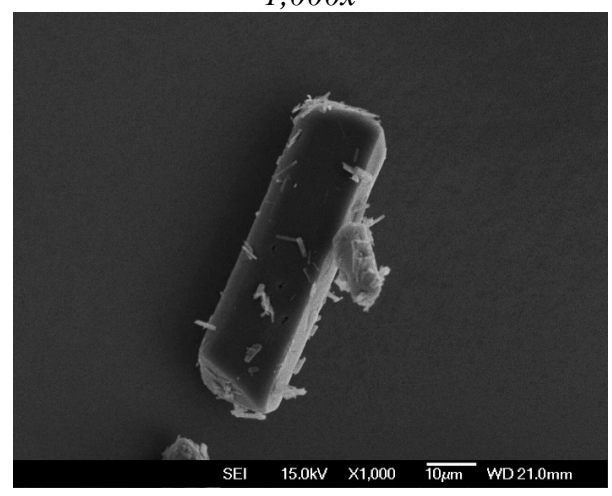

$300 x$

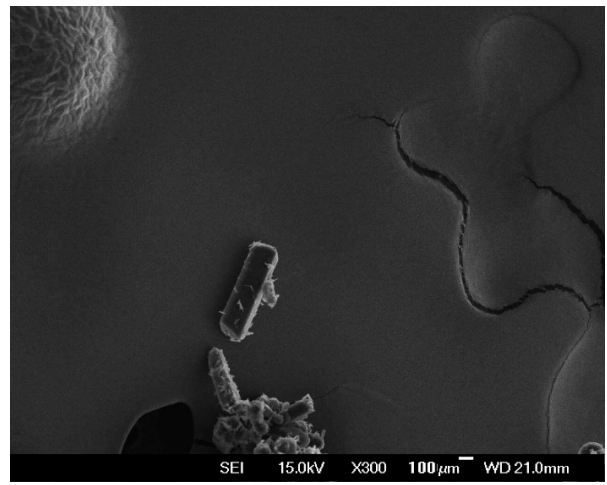

Fig. 6. Eight scanning electron microscope images of EuD 4 TEA crystallites made with europium acetate. The magnification factor used for the scan is shown above each image. A $12 \mathrm{~nm}$ gold layer was deposited on each sample before analysis. 


\section{$10,000 x$}

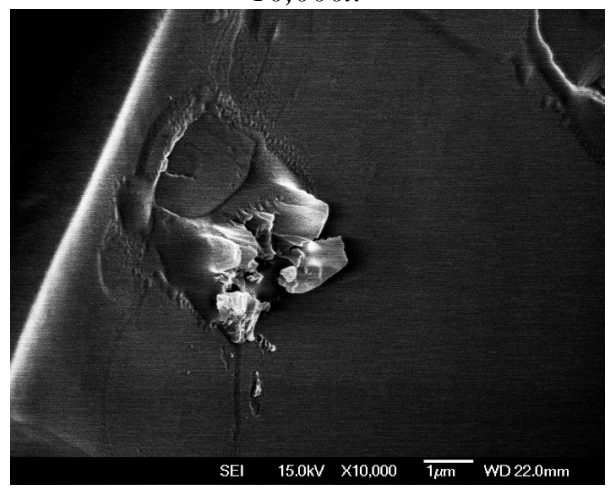

$10,000 x$

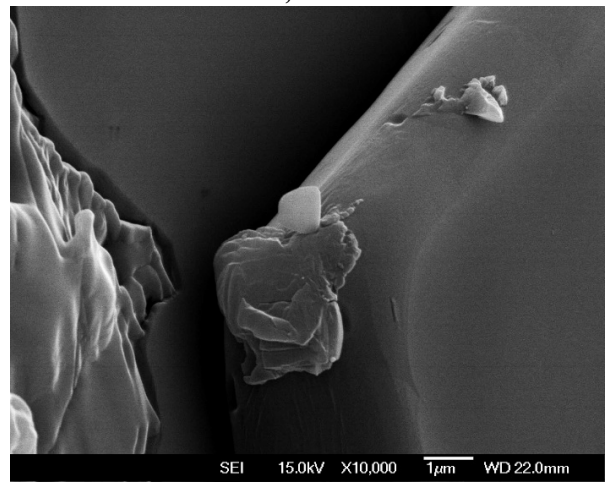

$1,000 x$

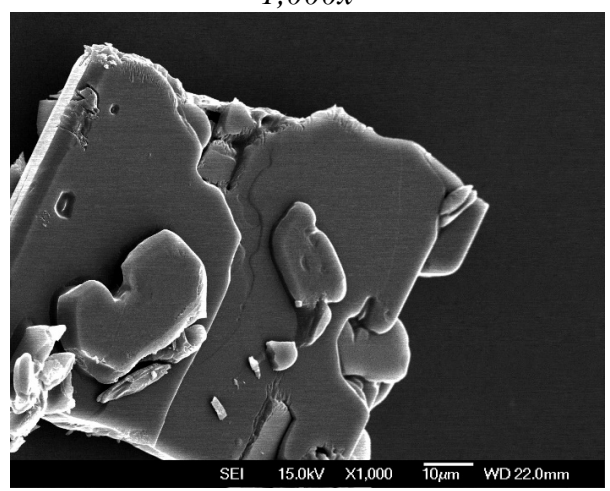

$100 x$

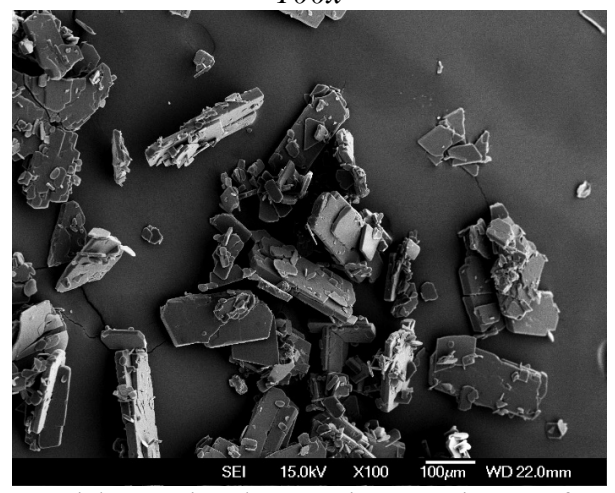

$5.000 x$

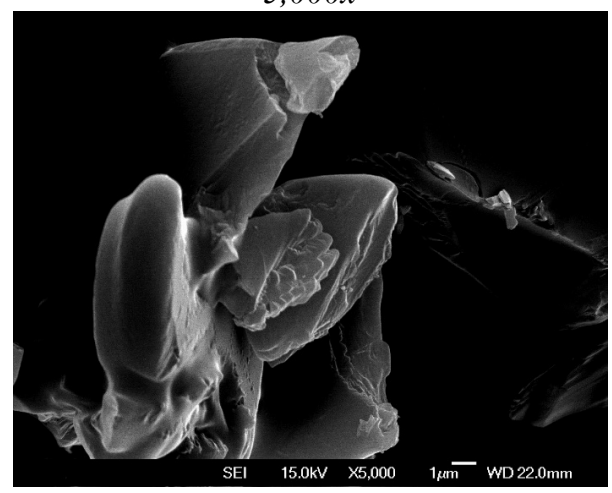

$1,000 x$

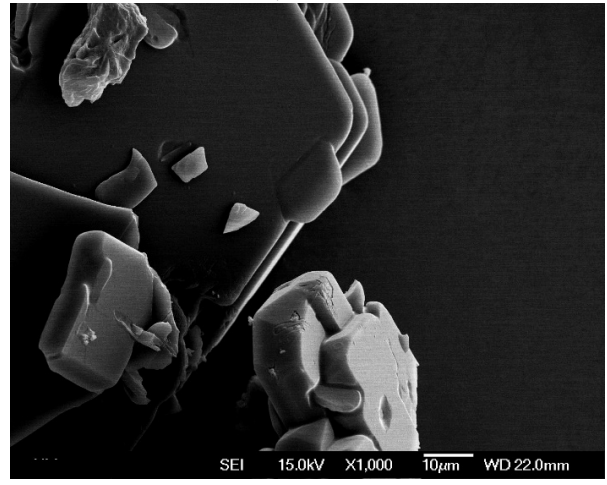

$1,000 x$

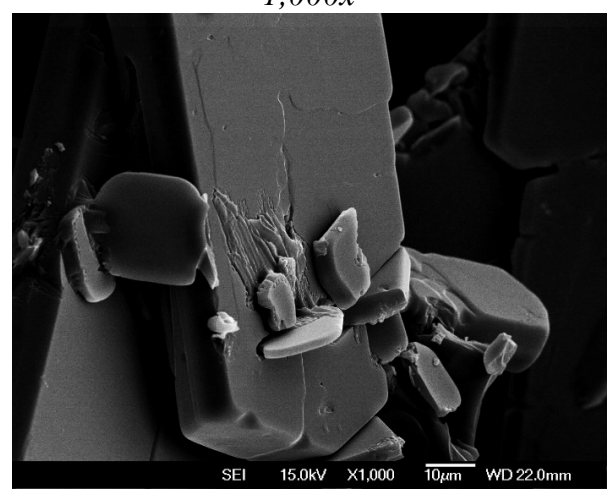

$300 x$

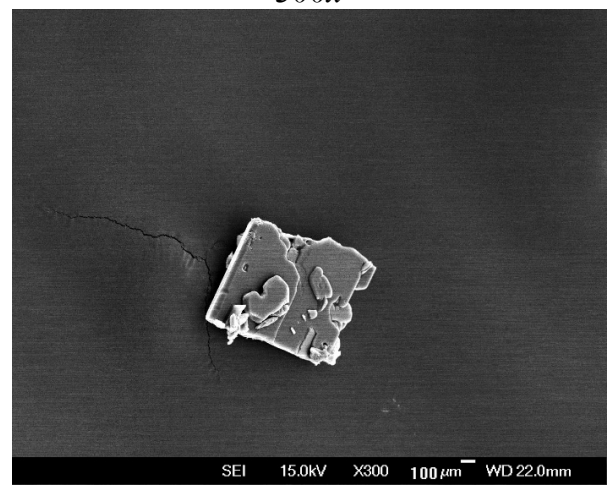

SEI $\quad 15.0 \mathrm{kV} \quad \times 300 \quad 100 \mathrm{\mu m}^{-}$WD $22.0 \mathrm{~mm}$

Fig. 7. Eight scanning electron microscope images of $\mathrm{EuD}_{4} \mathrm{TEA}$ crystallites made with europium nitrate. The magnification factor used for the scan is shown above each image. A $12 \mathrm{~nm}$ gold layer was deposited on each sample before analysis. 
The measured photoluminescent (PL) and triboluminescent emission spectra for $\mathrm{EuD}_{4} \mathrm{TEA}$ are shown in Figs. 8 and 9, respectively. Notice that the PL and TL excited emissions for both the acetate and nitrate precursor samples have the same peak wavelengths and are very bright. The spectra indicate that the luminescence from $\mathrm{EuD}_{4} \mathrm{TEA}$ originates from the typical $\mathrm{Eu}^{3+}$ centered transitions from the ${ }^{5} \mathrm{D}_{0}$ levels to the lower ${ }^{7} \mathrm{~F}_{0-4}$ levels [21-23]. The main emission occurred from the ${ }^{5} \mathrm{D}_{0} \rightarrow{ }^{7} \mathrm{~F}_{2}$ transitions.

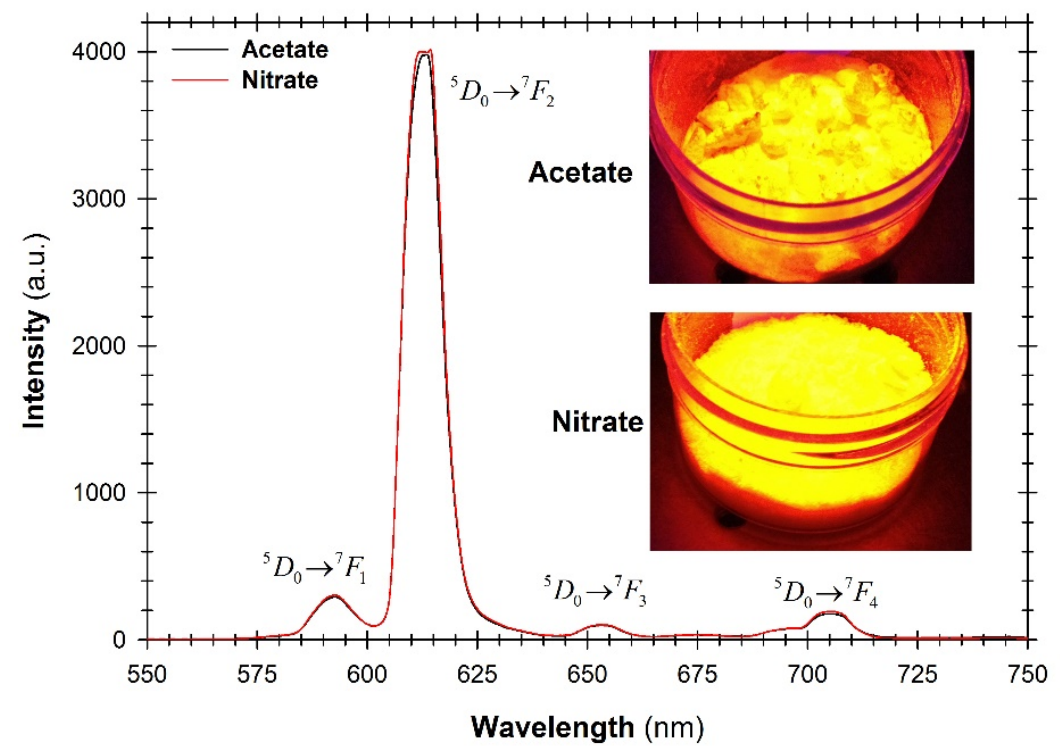

Fig. 8. Photoluminescent emission spectrum of EuD4TEA synthesized with europium acetate and nitrate precursors. The inset pictures show the PL emitted from the samples under the UV light.

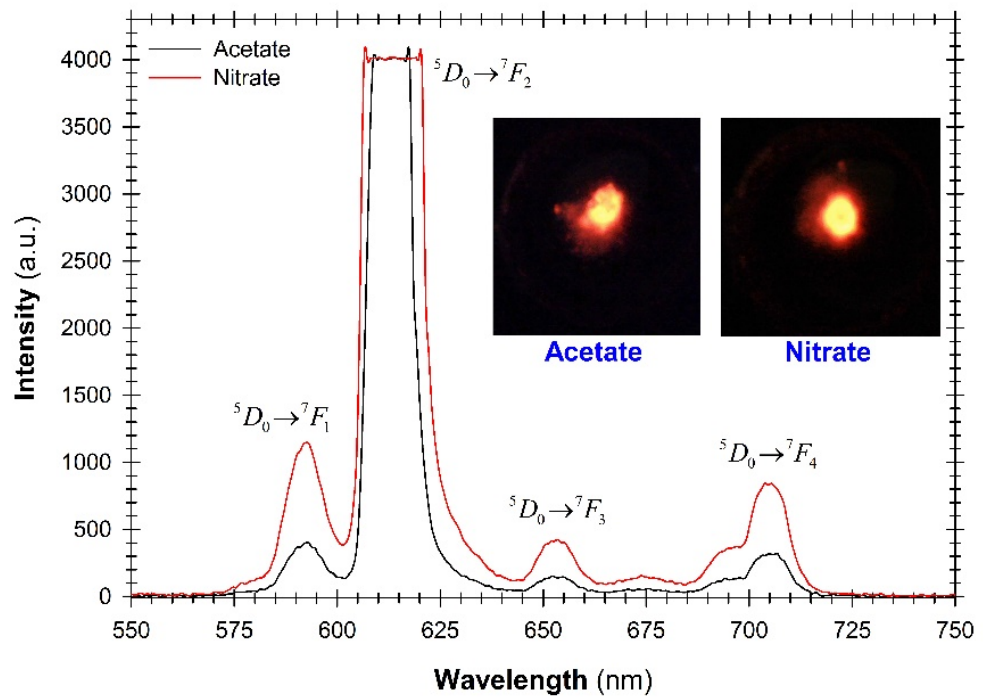

Fig. 9. Triboluminescent emission spectrum of $\mathrm{EuD}_{4} \mathrm{TEA}$ synthesized with europium acetate and nitrate precursors. The inset pictures show the TL emitted upon impact.

\section{Conclusions}

In order to reduce cost, the authors wanted to try using europium acetate to synthesize $\mathrm{EuD}_{4} \mathrm{TEA}$. It was found that the triboluminescent yield for $\mathrm{EuD}_{4} \mathrm{TEA}$ made with europium acetate was approximately half of what was measured for the sample made with europium nitrate. In addition, the EuD 4 TEA precursor crystals produced with the acetate are cloudy and only emit luminescence near the edges. Emission peaks from both the acetate and nitrate precursor samples have the same peak wavelengths, when excited by both PL and TL. The spectra indicate that the 
luminescence from $\mathrm{EuD}_{4} \mathrm{TEA}$ originates from the typical $\mathrm{Eu}^{3+}$ centered transitions from the ${ }^{5} \mathrm{D}_{0}$ levels to the lower ${ }^{7} \mathrm{~F}_{0-4}$ levels. The main emission occurred from the ${ }^{5} \mathrm{D}_{0} \rightarrow{ }^{7} \mathrm{~F}_{2}$ transitions. As a result, the use of europium acetate to synthesize EuD4TEA using ethyl alcohol is not practical, even with its reduced cost. Additional research needs to be completed to further quantify the best precursor materials for synthesizing EuD4TEA.

\section{Acknowledgements}

Funding for this research was provided by the Louisiana Space Grant Consortium. The authors would like to thank former physics students Jacque Meche, Stephen Williams, Colin McRae, and Lewis Baltz for assisting with this research.

\section{References}

[1] A.J. Walton, Triboluminescence, Adv. Phys. 26 (1977) 887-948. doi:10.1080/00018737700101483.

[2] R.S. Fontenot, K.N. Bhat, W.A. Hollerman, M.D. Aggarwal, Triboluminescent materials for smart sensors, Mater. Today. 14 (2011) $292-$ 293. doi:10.1016/S1369-7021(11)70147-X.

[3] R.S. Fontenot, W.A. Hollerman, B.M. Broussard, M.S. Steuart, Using Triboluminescent Impacts of ZnS: Mn as an Impact Detection Sensor for Spacecraft, in: G. Song, R.B. Malla (Eds.), Earth Sp. 2010, American Society of Civil Engineers, Honolulu, Hawaii, 2010: pp. 2560-2567. doi:doi:10.1061/41096(366)239.

[4] S.M. Goedeke, S.W. Allison, F.N. Womack, N.P. Bergeron, W.A. Hollerman, Triboluminescence and its application to space-based damage sensors, Oak Ridge, TN, 2001. http://www.ornl.gov/ webworks/cppr/y2001/pres/118019.pdf.

[5] W.A. Hollerman, S.M. Goedeke, N.P. Bergeron, R.J. Moore, S.W. Allison, L.A. Lewis, Emission spectra from ZnS:Mn due to low velocity impacts, in: E.W. Taylor (Ed.), Photonics Sp. Environ. X, SPIE, San Diego, CA, USA, 2005: p. 58970F-10. doi:10.1117/12.613570.

[6] D.O. Olawale, T. Dickens, W.G. Sullivan, O.I. Okoli, J.O. Sobanjo, B. Wang, Progress in triboluminescence-based smart optical sensor system, J. Lumin. 131 (2011) 1407-1418. doi:10.1016/j.jlumin.2011.03.015.

[7] I.C. Sage, G. Bourhill, Triboluminescent materials for structural damage monitoring, J. Mater. Chem. 11 (2001) $231-245$. doi:10.1039/b007029g.

[8] I.C. Sage, R. Badcock, L. Humberstone, N.J. Geddes, M. Kemp, G. Bourhill, Triboluminescent damage sensors, Smart Mater. Struct. 8 (1999) 504. doi:10.1088/0964-1726/8/4/308.

[9] I.C. Sage, L. Humberstone, I. Oswald, P. Lloyd, G. Bourhill, Getting light through black composites : embedded triboluminescent structural damage sensors, Smart Mater. Struct. 10 (2001) 332-337. doi:10.1088/0964-1726/10/2/320.

[10] C.N. Xu, T. Watanabe, M. Akiyama, X.G. Zheng, Artificial skin to sense mechanical stress by visible light emission, Appl. Phys. Lett. 74 (1999) 1236. doi:10.1063/1.123510.

[11] C.N. Xu, X.G. Zheng, M. Akiyama, K. Nonaka, T. Watanabe, Dynamic visualization of stress distribution by mechanoluminescence image, Appl. Phys. Lett. 76 (2000) 179. doi:10.1063/1.125695.

[12] C.S. Yoo, H.B. Radousky, N.C. Holmes, N.M. Edelstein, Luminescence of $\mathrm{Sm}^{2+}$ ions as a probe of pressure-induced phase transitions in $\mathrm{SrF}^{2}$, Phys. Rev. B. 44 (1991) 830-833. doi:10.1103/PhysRevB.44.830.

[13] J.I. Zink, Triboluminescence, Acc. Chem. Res. 11 (1978) 289-295. doi:10.1021/ar50128a001.

[14] I.C. Sage, N.J. Geddes, Triboluminescent damage sensors, 5905260, 1999.

[15] C.R. Hurt, N. Mcavoy, S. Bjorklund, N. Flipescu, High Intensity Triboluminescence in Europium Tetrakis (Dibenzoylmethide)triethylammonium, Nature. 212 (1966) 179-180. doi:10.1038/212179b0.

[16] K.N. Bhat, R.S. Fontenot, W.A. Hollerman, M.D. Aggarwal, Triboluminescent Research Review of Europium Dibenzoylmethide Triethylammonium (EuD4TEA) and Related Materials, Int. J. Chem. 1 (2012) 100-118. http://www.ijbb.in/index.php/ijc/article/view/58.

[17] R.S. Fontenot, W.A. Hollerman, M.D. Aggarwal, K.N. Bhat, S.M. Goedeke, A versatile low-cost laboratory apparatus for testing triboluminescent materials, Measurement. 45 (2012) 431-436. doi:10.1016/j.measurement.2011.10.031.

[18] R.S. Fontenot, K.N. Bhat, C.A. Owens, W.A. Hollerman, M.D. Aggarwal, Effects of added dibutyl phosphate on the luminescent properties of Europium Tetrakis Dibenzoylmethide Triethylammonium, J. Lumin. $156 \quad(2015) \quad 428-434$. doi:10.1016/j.jlumin.2014.10.026.

[19] N. Takada, J. Peng, N. Minami, Relaxation behavior of electroluminescence from europium complex light emitting diodes, Synth. Met. 121 (2001) 1745-1746. http://www.sciencedirect.com/science/article/pii/S0379677900006147.

[20] W.A. Hollerman, R.S. Fontenot, K.N. Bhat, M.D. Aggarwal, Measuring the Process Variability in Triboluminescence Emission Yield for EuD4TEA, Metall. Mater. Trans. A. 43 (2012) 4200-4203. doi:10.1007/s11661-012-1202-9.

[21] W.T. Carnall, P.R. Fields, K. Rajnak, Electronic Energy Levels of the Trivalent Lanthanide Aquo Ions. IV. Eu[sup $3+$ ], J. Chem. Phys. 49 (1968) 4450-4455. doi:10.1063/1.1669896.

[22] R.S. Fontenot, W.A. Hollerman, K.N. Bhat, M.D. Aggarwal, Synthesis and Characterization of Highly Triboluminescent Doped Europium Tetrakis Compounds, J. Lumin. 132 (2012) 1812-1818. doi:10.1016/j.jlumin.2012.02.027.

[23] R.S. Fontenot, K.N. Bhat, W.A. Hollerman, M.D. Aggarwal, K.M. Nguyen, Comparison of the triboluminescent yield and decay time for europium dibenzoylmethide triethylammonium synthesized using different solvents, CrystEngComm. 14 (2012) $1382-1386$. doi:10.1039/C2CE06277A. 